\title{
Electric field on nucleus in solids and interaction of CP-violating nuclear electric dipole moment with phonons
}

\author{
V. V. Flambaum and I. B. Samsonov \\ School of Physics, University of New South Wales, Sydney 2052, Australia
}

(Received 9 December 2019; accepted 24 March 2020; published 14 April 2020)

\begin{abstract}
In atoms and molecules, electrons screen the nucleus from the external electric field. However, if the frequency of the electric field reaches the energy of atomic or molecular transition, the electric field at the nucleus may be resonantly enhanced by many orders in magnitude. In this paper, we study the mechanisms of screening or enhancement of electric field acting on the nuclei in solid states. We show that in dielectric crystals the phonon oscillations of the lattice play a crucial role for determining the electric field on nuclei within the $\mathrm{MHz}-\mathrm{THz}$ region. As an application, we propose an experimental scheme for measuring the $\mathrm{CP}$-violating nuclear electric dipole moment in a solid state based on the coherent excitation of acoustic phonons.
\end{abstract}

DOI: 10.1103/PhysRevResearch.2.023042

\section{INTRODUCTION}

According to Schiff's theorem [1], atomic electrons screen the nucleus from the external electric field. In particular, a static electric field is completely screened inside neutral atoms and molecules thus creating complications in the measurements of the $\mathrm{CP}$-violating nuclear electric dipole moment (EDM). However, in real atoms and molecules this screening is incomplete due to the magnetic moment effect [1,2]. Moreover, the atomic EDM can be generated by the nuclear Schiff moment and magnetic quadrupole moment [3-5].

In ions and charged molecules the Schiff screening is incomplete [6,7], but charged particles accelerate in the electric field and quickly escape. Therefore, EDM measuring experiments with ions require special configurations of the electric and magnetic fields which trap the charged particles [8].

Another interesting possibility to circumvent Schiff's screening is to apply an oscillating electric field. As is demonstrated in the recent paper [9], the oscillating electric field inside atoms is screened only partly, and the suppression factor is proportional to the dynamic atomic polarizability. From the physical point of view, we understand this result as a lag in the displacement of the electron cloud in the changing electric field. However, when the frequency of the electric field reaches one of the energy levels of atomic transitions, the electric field at the nucleus may be enhanced by many orders in magnitude [10].

In molecules, the pattern of screening or resonant enhancement of the oscillating electric field is much more complex owing to the presence of rotational and vibrational states in addition to the electronic states [11]. Since the energies of

Published by the American Physical Society under the terms of the Creative Commons Attribution 4.0 International license. Further distribution of this work must maintain attribution to the author(s) and the published article's title, journal citation, and DOI. rovibrational states are lower than the ones for electronic states, even the fields in the microwave region may strongly interact with nuclei.

This paper is devoted to the extension of our previous results for atoms $[9,10]$ and molecules [11] to the case of solid states. On the one hand, a crystal can be viewed as a molecule composed of a large number of atoms, and the general consideration developed in [11] is applicable. However, the general formulas in [11] do not allow us to give specific numerical estimates as one needs to know the exact wave functions which take into account the positions of all atoms in the solid state including all excitations. On the other hand, in dielectrics and semiconductors, the electric field inside the solid state is well understood through the electric permittivity $\varepsilon=(1+\chi) \varepsilon_{0}$, where $\chi$ is the electric susceptibility and $\varepsilon_{0}$ is the vacuum permittivity. Indeed, the electric field $\mathbf{E}$ creates the polarization in the medium $\mathbf{P}=\varepsilon_{0} \chi \mathbf{E}$ which reduces the electric field in the solid by the factor of $\varepsilon_{\mathrm{r}}=1+\chi$. However, this electric field is macroscopic while we need to know the microscopic electric field acting on each nucleus in the solid state.

In this paper, we consider dielectric crystals with no free electrons to avoid strong surface screening of the electric field. As we will demonstrate, it is possible to estimate the electric field at each nucleus when the phonon excitation of the lattice is known. For our estimates, it will be sufficient to consider phonon lattice oscillations semiclassically. Moreover, we will restrict ourselves to the case of a harmonic crystal assuming that anharmonic corrections are subleading.

To sketch the main idea, consider an ideal crystal in a state with no phonons such that the atoms in the lattice do not vibrate. This means that each nucleus sits in the minimum of the electrostatic potential of the lattice such that the electric field on each nucleus is vanishing. Assume now that a phonon is excited with a given wave vector $\mathbf{q}$ and frequency $\omega$. Then, for small perturbations, each atom (or ion) in the lattice oscillates around its equilibrium position harmonically, $\mathbf{u}(t)=\mathbf{u}_{0} \cos \omega t$, where $\mathbf{u}$ is the deviation of the given atom 
from its equilibrium position. Discarding the magnetic interaction, one can immediately deduce that each nucleus with mass $m$ and charge $Z e$ accelerates in the electric field $\mathbf{E}_{\text {nucl }}=\frac{m}{Z e} \ddot{\mathbf{u}}$.

Normally, at nonzero temperature, the lattice contains a large number of phonons of all allowed frequencies and polarizations. In this case, the oscillation of each atom in the lattice is chaotic, with no preferred direction, and the electric field at the nucleus averages to zero. However, modern technology allows one to coherently create a given number of phonons in the solid state with fixed polarization and frequency (see, e.g., $[12,13])$. Thus, when the pattern of the phonons in the solid state is known, one can deduce the value of the oscillating electric field acting on each nucleus. This technique may be useful in the experiments aiming to study properties of atomic nuclei and, in particular, the nuclear EDM.

In this paper, unless other units are explicitly specified, we use natural units, in which $\hbar=c=1$, where $c$ is the speed of light.

The rest of the paper is organized as follows. In the next section we derive analytical expressions for the electric field at the nucleus induced by acoustic and optical phonons in solids. In Sec. III, we numerically estimate the largest magnitude of the electric field at the nucleus in solid xenon created by coherent acoustic phonons. We also consider the optical phonons in a sodium chlorine crystal and show that they may enhance the external electric field acting on the nucleus when the frequency of this field is in resonance with the frequency of transverse optical phonons. In Sec. IV, we present a theoretical idea of how the coherently excited acoustic phonons may be used in experiments aimed at measuring the permanent nuclear EDM. In the last section we summarize and briefly discuss the obtained results.

\section{ELECTRIC FIELD ON THE NUCLEUS DUE TO PHONON LATTICE OSCILLATIONS}

In this section, we derive analytic expressions for the electric field acting on nuclei in crystals due to acoustic and optical phonons. In our derivation we restrict ourselves to the theory of harmonic crystals, leaving the analysis of anharmonic corrections for further studies.

\section{A. Electric field due to acoustic phonons}

Acoustic phonons with long wavelengths may be considered as sound waves in a continuous medium with a frequency $\omega$ and a wave vector $\mathbf{q}$ :

$$
\mathbf{u}(\mathbf{r}, t)=\mathbf{u}_{0} \cos (\mathbf{q} \cdot \mathbf{r}-\omega t),
$$

where $\mathbf{u}_{0}$ is the amplitude. Consider, in particular, the oscillations of the atom at $\mathbf{r}=0$, in the direction $\mathbf{u}_{0}=\left(0,0, u_{0}\right)$, $u(t)=u_{0} \cos \omega t$. This oscillation reaches the maximum amplitude when the crystal is nearly melted or destroyed. Typically, this oscillation amplitude is of order 0.1 of interatomic distance $a[14]$ :

$$
u_{0, \max }=\eta a, \quad \eta \approx 0.1
$$

Thus, in the harmonic approximation, the largest oscillation of the atomic nucleus due to the given phonons reads

$$
u(t)=\eta a \cos \omega t .
$$

This motion is produced by the microscopic electric field acting on the nucleus:

$$
E_{\text {nucl }}=\frac{m}{Z e} \ddot{u}=-\frac{\eta a m \omega^{2}}{Z e} \cos \omega t,
$$

where $m$ is the mass of the nucleus and $Z e$ is its charge. Thus, the maximum amplitude of the electric field on the nucleus due to acoustic phonons reads

$$
E_{0}=-\frac{\eta a m \omega^{2}}{Z e}
$$

As we will show in Sec. III A, for low frequencies this electric field may be much stronger than the screened external electric field.

\section{B. Electric field due to optical phonons}

Let us consider a simple cubic lattice with two ions per unit cell. Let $\left(\mathbf{u}_{1}, m_{1}\right)$ and $\left(\mathbf{u}_{2}, m_{2}\right)$ be pairs of coordinates and masses of the two ions in the unit cell. To describe the oscillations around the common center of mass, it is convenient to introduce the phonon coordinate

$$
\mathbf{u}=\sqrt{m N}\left(\mathbf{u}_{1}-\mathbf{u}_{2}\right),
$$

where $m$ is the reduced mass, $m^{-1}=m_{1}^{-1}+m_{2}^{-1}$, and $N$ is the atom number density. In the external oscillating electric field $\mathbf{E}=\mathbf{E}_{0} \cos \omega t$ the dynamics of the variable $\mathbf{u}$ is described by the equation

$$
\ddot{\mathbf{u}}+\gamma \dot{\mathbf{u}}+\omega_{\mathrm{t}}^{2} \mathbf{u}=Z^{*} \mathbf{E}_{0} \cos \omega t,
$$

where $\gamma$ is the phonon inverse lifetime, $\omega_{\mathrm{t}}$ is the transverse optical phonon frequency, and $Z^{*}$ is the effective ion charge. The latter may be expressed via the dielectric constants of the solid state (see, e.g., [14]):

$$
Z^{*}=\omega_{\mathrm{t}} \sqrt{\frac{\varepsilon_{\mathrm{stat}}-\varepsilon_{\mathrm{opt}}}{4 \pi}} \frac{3}{\varepsilon_{\mathrm{opt}}+2},
$$

where $\varepsilon_{\text {stat }}$ and $\varepsilon_{\text {opt }}$ are the electric permittivity for static and high-frequency electric fields, respectively.

In the harmonic approximation, both $\omega_{\mathrm{t}}$ and $Z^{*}$ are constants. More generally, one can consider the phonon dynamics with anharmonic corrections, but this problem is beyond the scope of this paper.

The steady-state solution of Eq. (7) reads

$$
\mathbf{u}=\mathbf{u}_{0} \cos (\omega t+\phi),
$$

where

$$
\begin{gathered}
\mathbf{u}_{0}=-\frac{Z^{*} \mathbf{E}_{0}}{\sqrt{\left(\omega_{\mathrm{t}}^{2}-\omega^{2}\right)^{2}+(\omega \gamma)^{2}}}, \\
\phi=\arctan \frac{\omega \gamma}{\omega^{2}-\omega_{\mathrm{t}}^{2}} .
\end{gathered}
$$

The electric field acting on the ions can be found as

$$
\mathbf{E}_{\mathrm{ion}}=\frac{1}{q} \sqrt{\frac{m}{N}} \ddot{\mathbf{u}}=-\frac{\omega^{2}}{q} \sqrt{\frac{m}{N}} \mathbf{u}_{0} \cos (\omega t+\phi),
$$


where $q$ is the charge of the ion. The amplitude of this field is

$$
\begin{aligned}
\mathbf{E}_{\mathrm{ion}, 0} & =-\frac{\omega^{2}}{q} \sqrt{\frac{m}{N}} \mathbf{u}_{0} \\
& =\frac{Z^{*}}{q} \sqrt{\frac{m}{N}} \frac{\omega^{2}}{\sqrt{\left(\omega_{\mathrm{t}}^{2}-\omega^{2}\right)^{2}+(\omega \gamma)^{2}}} \mathbf{E}_{0} .
\end{aligned}
$$

When the frequency of the external electric field $\omega$ is far from the phonon resonance frequency $\omega_{\mathrm{t}}, \omega \ll \omega_{\mathrm{t}}$, Eq. (13) reduces to

$$
\mathbf{E}_{\mathrm{ion}, 0}=\frac{Z^{*}}{q} \sqrt{\frac{m}{N}} \frac{\omega^{2}}{\omega_{\mathrm{t}}^{2}-\omega^{2}} \mathbf{E}_{0} .
$$

In contrast, near resonance, $\omega \approx \omega_{\mathrm{t}},\left|\omega-\omega_{\mathrm{t}}\right| \ll \gamma$, the amplitude of the electric field amplifies:

$$
\mathbf{E}_{\mathrm{ion}, 0}=\frac{Z^{*}}{q} \sqrt{\frac{m}{N}} \frac{\omega_{\mathrm{t}}}{\gamma} \mathbf{E}_{0}
$$

Finally, we point out that the bound electrons of each ion further screen the electric field on the nucleus by the law [9]

$$
\mathbf{E}_{\text {nucl }}=\mathbf{E}_{\text {ion }}\left(\frac{q}{Z}-\alpha_{\text {ion }}(\omega) \frac{\omega^{2} m_{e}^{2}}{Z e^{2}}\right),
$$

where $\alpha_{\text {ion }}(\omega)$ is the atomic polarizability of the ion.

\section{NUMERICAL ESTIMATES}

In this section, we give numerical estimates of the electric field on nuclei induced by acoustic and optical phonons. For the acoustic phonons, we consider solid xenon because it may be further applied in nuclear EDM measurement experiments. The electric field due to the optical phonons is considered in the sodium chlorine crystal because its optical and dielectric properties are well represented in the literature.

\section{A. Electric field in solid xenon}

Let us consider ${ }^{129} \mathrm{Xe}$ noble gas in the solid state below the melting temperature $T_{\text {melt }}=161 \mathrm{~K}$. The nuclear magnetic dipole moment of this isotope is $\mu \approx-0.78 \mu_{N}$ [15], where $\mu_{N}$ is the nuclear magneton. In a strong magnetic field $B=10 \mathrm{~T}$, the nuclear spin Larmor precession frequency is $\omega=2 \mu B=2 \pi \times 119 \mathrm{MHz}=4.9 \times 10^{-7} \mathrm{eV}$. We will be interested in acoustic phonons in the xenon crystal with this frequency $\omega$.

The crucial assumption in our estimate will be that it is possible to coherently excite the phonons in the solid state with the given frequency and the wave vector. In particular, it is possible to use a pulsed laser with pulses modulated to a given frequency $\omega$ to coherently generate acoustic phonons (see, e.g., [12] for a review). Moreover, we point out that modern technology allows one to measure precisely the number of phonons created in the solid state [13]. Without going further into the details of these techniques we will assume that they may be applied to a solid xenon sample.

Given that the lattice parameter in the solid xenon is $a=$ $6.2 \AA$, the maximum electric field at the nucleus may be estimated from Eq. (5):

$$
E_{0, \max } \approx 0.9 \mathrm{~V} / \mathrm{m} \text {. }
$$

It is instructive to compare the electric field due to phonon lattice vibrations with the screening of the external electric field on the nucleus in an isolated Xe atom. According to [9], the electric field at the nucleus induced by the external electric field $E=E_{0} \cos (\omega t)$ is

$$
E_{\text {nucl }}=\frac{\tilde{\alpha} \tilde{\omega}^{2}}{Z} E \approx 1.6 \times 10^{-16} E,
$$

where $\tilde{\alpha}=27.3$ is the static atomic polarizability of xenon and $\tilde{\omega}=\omega a_{B} / e^{2} \approx 1.8 \times 10^{-8}$ is the energy in atomic units. Thus, for any reasonable laboratory electric field $E_{0}$ the electric field on the nucleus due to phonons (17) is much stronger than the external electric field screened by the atomic electrons (18).

\section{B. Electric field due to optical phonons in sodium chloride}

Dielectric and phonon properties of $\mathrm{NaCl}$ are well known (see, e.g., [14]). In particular, the electric permittivity for static and optical frequency electric fields is $\varepsilon_{\text {stat }}=5.9, \varepsilon_{\mathrm{opt}}=2.34$, and the transverse optical phonon resonant frequency is $\omega_{\mathrm{t}}=$ $0.02 \mathrm{eV}$. The phonon width $\gamma$ may be estimated as $\omega_{\mathrm{t}} / \gamma \approx$ 50 [16]. The reduced mass for the $\mathrm{NaCl}$ molecule is $m=$ $13.9 m_{p}$, and the atom number density is $N \approx 2 \times 10^{22} \mathrm{~cm}^{-3}$. According to Eq. (15), the electric field acting on each ion is

$$
E_{\text {ion }, 0} \approx 39 E_{0} .
$$

Given the electric field at the ion, the field at the nucleus may be obtained with Eq. (16). At the frequency $\omega=\omega_{\mathrm{t}}$ the last term in Eq. (16) is negligible. Then, for the Na ion $Z=11$ and $N_{e}=10$, and we have

$$
E_{\text {nucl }} \approx \frac{1}{11} E_{\text {ion }} \approx 3.5 E .
$$

Thus, the oscillating electric field in resonance with the transverse optical phonons not only reaches the nucleus, but may also be enhanced by the factor of 3.5 .

Note that, in Eq. (20), the electric field at the nucleus grows linearly with $E$ only for a weak external field when the harmonic description of phonons applies. We stress that the magnitude of the electric field cannot exceed the value (5) at which the lattice oscillations reach the maximum amplitude. For the $\mathrm{NaCl}$ crystal, this maximum electric field is of order $10^{7} \mathrm{~V} / \mathrm{m}$ at the frequency $\omega=\omega_{\mathrm{t}}=2 \pi \times 480 \mathrm{GHz}$.

Note also that if one considers the electric field off the resonance with the optical phonon frequency, Eq. (14) applies. In this case, instead of the resonance enhancement there is off-resonance suppression.

\section{APPLICATION: NUCLEAR EDM MEASUREMENT}

Recently, a few experiments have been proposed to measure nuclear and electron EDMs with solid-state samples [17-21]. It is expected that such experiments may have better sensitivity as compared to traditional EDM experiments on atoms and molecules in the vapor state or in beams and stored ions in traps. However, as was noticed in [19,22], one of the issues of the solid-state EDM experiments is that the oscillating strong electric field causes heating of the sample and thermal depolarization of spins. This problem does not 


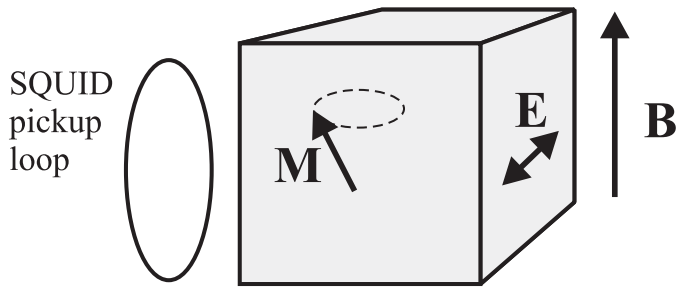

FIG. 1. Orientation of magnetic and electric fields in the solidstate EDM experiments. The external magnetic field $\mathbf{B}$ is created by a strong magnet while the electric field $\mathbf{E}$ is the microscopic electric field on nuclei created by coherent phonon lattice oscillations in the given direction. The interaction of the nuclear EDM with an oscillating electric field creates the transverse macroscopic magnetization $\mathbf{M}$ of the sample which may by detected by a superconducting quantum interference device magnetometer.

arise in the CASPEr experiment [22,23] aimed at the detection of the oscillating EDM induced by axion dark matter.

In this section, we will present an idea of how to measure the permanent nuclear EDM using the phonon excitations in solids instead of applying an external oscillating electric field. We will consider an experimental setup similar to the CASPEr experiment [22,23], but with important modifications needed to measure the permanent nuclear EDM. A very schematic design of this experiment with main emphasis on the orientation of electric and magnetic fields is shown in Fig. 1. Nuclear spins in this solid state are prepolarized by the external strong magnetic field $\mathbf{B}$. The magnitude of this field determines the Larmor precession frequency $\omega=2 \mu B$, where $\mu$ is the nuclear magnetic moment. Nuclear EDMs are also prepolarized by this field, because the nuclear EDM is parallel to the nuclear spin.

The oscillating electric field $E_{\text {nucl }}=E_{\text {nucl }, 0} \cos \omega t$ acting on the nuclei should be created in the orthogonal plane. We assume that this field is coherently created due to acoustic phonons as described in Sec. III A. The frequency of this field should match the Larmor precession frequency of nuclear spins. In this case, the NMR-like interaction of the oscillating electric field with the nuclear EDM $d_{N}$ creates the macroscopic magnetization $M$ of the sample in the direction orthogonal to both $\mathbf{B}$ and $\mathbf{E}_{\text {nucl }}$. For a small $\left(d_{N} t\right)$ this magnetization grows linearly with time:

$$
M(t) \approx N \mu E_{\text {nucl }} d_{N} t \sin (2 \mu B t),
$$

where $N$ is the number density of nuclear spins, $\mu$ is the nuclear magnetic moment, and $B$ is the external magnetic field. This magnetization may be detected by a magnetometer like a superconducting quantum interference device (SQUID).

For the solid state, it is convenient to use solid xenon due to a number of reasons.

(i) It has odd stable isotopes ${ }^{129} \mathrm{Xe}$ and ${ }^{131} \mathrm{Xe}$ with nuclear spins $1 / 2$ and $3 / 2$, respectively, which are often used in the EDM measurements with NMR technology [24-26]. Here, for simplicity, we consider ${ }^{129} \mathrm{Xe}$ only although other options are possible.

(ii) The melting temperature of xenon is high compared to other noble gases. (iii) In solid state, xenon possesses a cubic lattice which makes it simple for theoretical studies.

(iv) Lastly, since all electron shells are closed, only the nuclear EDM interacting with the electric field may be responsible for the macroscopic magnetization of the sample.

For solid xenon, the parameters in Eq. (21) are $N=1.66 \times$ $10^{22} \mathrm{~cm}^{-3}, \mu=-0.78 \mu_{N}$, and $2 \mu B=4.9 \times 10^{-7} \mathrm{eV}$ for $B=10 \mathrm{~T}$. The maximum electric field at the nucleus due to phonon lattice oscillations is estimated in Eq. (17), $E_{\text {nucl }} \approx$ $0.9 \mathrm{~V} / \mathrm{m}$.

The macroscopic magnetization of the sample may be detected with a magnetometer. We assume a SQUID magnetometer with sensitivity $10^{-15} \mathrm{~T} / \sqrt{\mathrm{Hz}}$. Assuming that the measurement time may be of order $t \sim 1000 \mathrm{~s}$ (see, e.g., [27]), the solid-state EDM experiment may detect the permanent nuclear EDM of order

$$
d_{N} \approx 1.2 \times 10^{-22} e \mathrm{~cm} .
$$

The above estimate is very close to current experimental limits on the nuclear EDM. The best limit on the atomic EDM in xenon was obtained in [24]: $\left|d_{\mathrm{Xe}}\right|<1.5 \times 10^{-27} e \mathrm{~cm}$. According to [2], in xenon, the nuclear EDM contributes to the atomic EDM as $d_{\mathrm{Xe}}=4.4 \times 10^{-6} d_{N}$. Thus, the xenon EDM experiment puts the following limit on the nuclear EDM in xenon:

$$
\left|d_{N}\right|<2.5 \times 10^{-22} e \mathrm{~cm} .
$$

As a result, it may be promising to develop an experimental technique for measuring the nuclear EDM using phonon lattice oscillations.

We stress that Eq. (22) represents a very crude estimate of sensitivity of an experiment aimed at measuring the nuclear EDM in solids using lattice oscillations. In a more accurate estimate one is to analyze the signal-to-noise ratio by taking into account different noise sources in realistic experiments. This analysis will be done elsewhere. Here we only present the theoretical idea of possible application of coherent phonon excitations in solids to the nuclear EDM measurement. We point out that our proposal allows for direct nuclear EDM measurement which is free from the problem of Schiff's screening arising in classic EDM experiments with paramagnetic atomic and molecular systems $[26,28-30]$. These experiments aim to measure the atomic EDM which may be, in particular, induced by the nuclear Schiff moments [3,4].

Finally, we point out that the experimental technique described in this section may be applied to ionic crystals. In this case, it might be possible to measure the EDMs of ions induced by the nuclear Schiff moment, magnetic quadrupole moment, or electron EDM. The electric field on the nucleus induced by the phonon lattice oscillations may also be used to measure the oscillating nuclear EDM induced by the axion dark matter. The possibility of this measurement for atoms and molecules with the use of an external oscillating electric field was discussed in [31].

\section{SUMMARY}

In this paper, we estimated the magnitude of the electric field induced on the atomic nuclei by phonon lattice oscillations in solid states. In these oscillations, the maximum 
deviation of atoms from their equilibrium positions is typically of the order of one-tenth of the interatomic distance that corresponds to the maximum magnitude of the electric field acting on the nuclei as in Eq. (5). If the atoms oscillate randomly due to the thermal motion, this field averages out in time. However, if the acoustic phonons can be created coherently in all atoms in the solid state with a given wave vector and polarization, this electric field becomes significant. As we demonstrated, for low frequencies (characteristic of the acoustic phonons) this electric field acting on atomic nuclei due to phonon lattice oscillation is much stronger than the external electric field screened by the atomic electrons. In particular, in solid xenon, this field is of order $1 \mathrm{~V} / \mathrm{m}$ at frequency $119 \mathrm{MHz}$.

The crucial assumption in our estimate is that the acoustic phonons may be excited coherently with a given wave vector and polarization. This assumption is based on advances in phonon generation and counting (see, e.g., $[12,13])$. As we advocate in Sec. IV, this technique may be applied to measure the nuclear EDM. We expect that possible NMR-like experiments based on the phonon lattice oscillations may be sensitive to the nuclear EDM of order $10^{-22} e \mathrm{~cm}$. This sensitivity is very close to the current constraint on the nuclear EDM (23) arising from the recent atomic EDM measurement in xenon [24]. Thus, it is tempting to develop an experimental technique for measuring the nuclear EDM with phonon lattice oscillations in solids.

We also estimated the electric field at the nucleus due to optical phonons which are present in many ionic crystals. In contrast to the acoustic phonons, the optical ones interact directly with the external electric field. When this electric field is in resonance with the frequency of transverse optical phonons, it may be enhanced on the nucleus. Possible experimental applications of the electric field on the nucleus induced by the optical phonons will be discussed elsewhere.

\section{ACKNOWLEDGMENTS}

This work is supported by Australian Research Council Grant No. DP150101405 and by a Gutenberg Fellowship. I.B.S. is grateful to H. B. Tran Tan for useful comments.
[1] L. I. Schiff, Measurability of nuclear electric dipole moments, Phys. Rev. 132, 2194 (1963).

[2] S. G. Porsev, J. S. M. Ginges, and V. V. Flambaum, Atomic electric dipole moment induced by the nuclear electric dipole moment: The magnetic moment effect, Phys. Rev. A 83, 042507 (2011).

[3] O. P. Sushkov, V. V. Flambaum, and I. B. Khriplovich, Possibility of investigating $\mathrm{P}$ - and T-odd nuclear forces in atomic and molecular experiments, Zh. Exp. Teor. Fiz. 87, 1521 (1984) [Sov. Phys. JETP 60, 873 (1984)].

[4] V. V. Flambaum, I. B. Khriplovich, and O. P. Sushkov, On the P- and T-nonconserving nuclear moments, Nucl. Phys. A 449, 750 (1986).

[5] V. V. Flambaum, Spin hedgehog and collective magnetic quadrupole moments induced by parity and time invariance violating interaction, Phys. Lett. B 320, 211 (1994).

[6] V. A. Dzuba, V. V. Flambaum, P. G. Silvestrov, and O. P. Sushkov, Shielding of an external electric field in atoms, Phys. Lett. A 118, 177 (1986).

[7] V. V. Flambaum and A. Kozlov, Extension of the Schiff theorem to ions and molecules, Phys. Rev. A 85, 022505 (2012).

[8] W. B. Cairncross, D. N. Gresh, M. Grau, K. C. Cossel, T. S. Roussy, Y. Ni, Y. Zhou, J. Ye, and E. A. Cornell, Precision Measurement of the Electron's Electric Dipole Moment Using Trapped Molecular Ions, Phys. Rev. Lett. 119, 153001 (2017).

[9] V. V. Flambaum, Shielding of an external oscillating electric field inside atoms, Phys. Rev. A 98, 043408 (2018).

[10] V. V. Flambaum and I. B. Samsonov, Resonant enhancement of an oscillating electric field in an atom, Phys. Rev. A 98, 053437 (2018).

[11] H. B. T. Tan, V. V. Flambaum, and I. B. Samsonov, Screening and enhancement of an oscillating electric field in molecules, Phys. Rev. A 99, 013430 (2019).

[12] P. Ruello and V. E. Gusev, Physical mechanisms of coherent acoustic phonons generation by ultrafast laser action, Ultrasonics 56, 21 (2015).
[13] L. R. Sletten, B. A. Moores, J. J. Viennot, and K. W. Lehnert, Resolving Phonon Fock States in a Multimode Cavity with a Double-Slit Qubit, Phys. Rev. X 9, 021056 (2019).

[14] N. W. Ashcroft and N. D. Mermin, Solid State Physics (Saunders, Philadelphia, 1976).

[15] W. Makulski, ${ }^{129} \mathrm{Xe}$ and ${ }^{131} \mathrm{Xe}$ nuclear magnetic dipole moments from gas phase NMR spectra, Magn. Reson. Chem. 53, 273 (2015).

[16] G. Raunio, Phonon widths in $\mathrm{NaCl}, \mathrm{KCl}$, and $\mathrm{RbCl}$ from neutron measurements, Phys. Status Solidi 35, 299 (1969).

[17] S. K. Lamoreaux, Solid-state systems for the electron electric dipole moment and other fundamental measurements, Phys. Rev. A 66, 022109 (2002).

[18] T. N. Mukhamedjanov and O. P. Sushkov, Suggested search for ${ }^{207} \mathrm{~Pb}$ nuclear Schiff moment in $\mathrm{PbTiO}_{3}$ ferroelectric, Phys. Rev. A 72, 034501 (2005).

[19] D. Budker, S. K. Lamoreaux, A. O. Sushkov, and O. P. Sushkov, Sensitivity of condensed-matter P- and T-violation experiments, Phys. Rev. A 73, 022107 (2006).

[20] A. O. Sushkov, S. Eckel, and S. K. Lamoreaux, Prospects for an electron electric-dipole-moment search with ferroelectric (Eu, Ba) $\mathrm{TiO}_{3}$ ceramics, Phys. Rev. A 81, 022104 (2010).

[21] K. Z. Rushchanskii et al., A multiferroic material to search for the permanent electric dipole moment of the electron, Nat. Mater. 9, 649 (2010).

[22] D. Budker, P. W. Graham, M. Ledbetter, S. Rajendran, and A. O. Sushkov, Proposal for a Cosmic Axion Spin Precession Experiment (CASPEr), Phys. Rev. X 4, 021030 (2014).

[23] D. F. Jackson Kimball et al., Overview of the cosmic axion spin precession experiment (CASPEr), arXiv:1711.08999.

[24] F. Allmendinger, I. Engin, W. Heil, S. Karpuk, H.-J. Krause, B. Niederänder, A. Offenhäusser, M. Repetto, U. Schmidt, and S. Zimmer, Measurement of the permanent electric dipole moment of the ${ }^{129} \mathrm{Xe}$ atom, Phys. Rev. A 100, 022505 (2019). 
[25] N. Sachdeva et al., A new measurement of the permanent electric dipole moment of ${ }^{129} \mathrm{Xe}$ using ${ }^{3} \mathrm{He}$ comagnetometry and SQUID detection, arXiv:1902.02864v1.

[26] M. A. Rosenberry and T. E. Chupp, Atomic Electric Dipole Moment Measurement Using Spin Exchange Pumped Masers of ${ }^{129} \mathrm{Xe}$ and ${ }^{3} \mathrm{He}$, Phys. Rev. Lett. 86, 22 (2001).

[27] M. V. Romalis and M. P. Ledbetter, Transverse Spin Relaxation in Liquid ${ }^{129} \mathrm{Xe}$ in the Presence of Large Dipolar Fields, Phys. Rev. Lett. 87, 067601 (2001).

[28] B. Graner, Y. Chen, E. G. Lindahl, and B. R. Heckel, Reduced Limit on the Permanent Electric Dipole Moment of ${ }^{199} \mathrm{Hg}$, Phys. Rev. Lett. 116, 161601 (2016); Erratum:
Reduced Limit on the Permanent Electric Dipole Moment of ${ }^{199} \mathrm{Hg}$ [Phys. Rev. Lett. 116, 161601 (2016)] 119, 119901(E) (2017).

[29] R. H. Parker et al., First Measurement of the Atomic Electric Dipole Moment of ${ }^{225}$ Ra, Phys. Rev. Lett. 114, 233002 (2015).

[30] D. Cho, K. Sangster, and E. A. Hinds, Search for time-reversalsymmetry violation in thallium fluoride using a jet source, Phys. Rev. A 44, 2783 (1991).

[31] V. V. Flambaum, H. B. Tran Tan, D. Budker and A. Wickenbrock, Atomic and molecular transitions induced by axions via oscillating nuclear moments, arXiv:1910.07705. 